\title{
Internacionalización de cooperativas agrarias. El modelo de la empresa española Covap S.C.A.*
}

\author{
Sánchez Cañizares, Sandra $\mathbf{M}^{a * *}$ \\ Fuentes García, Fernando J.*** \\ Santos Roldán, Luna $\mathbf{M}^{\mathrm{a} * * * *}$
}

\section{Resumen}

En este trabajo se realiza un análisis de la situación de la empresa cooperativa de cara al proceso de internacionalización, particularizando el estudio al caso de las cooperativas agrarias por su destacado papel en el marco económico de la Unión Europea. Se aplica la metodología de análisis de casos para revisar la situación de la Sociedad Cooperativa Agraria Valle de los Pedroches (COVAP S.C.A), situada en Andalucía (España), que se presenta como ejemplo de cómo una pequeña cooperativa de ganaderos a sus inicios, ha pasado a convertirse en sus 50 años de vida en una de las cooperativas agrarias de primer grado, líder en facturación en España, y a llevar sus productos a más de 23 países entre los que se encuentran mercados tan exigentes como es Estados Unidos. Las conclusiones muestran, por un lado, la exigencia y el reto que supone para las cooperativas agrarias el proceso de internacionalización aún con las dificultades que supone en este tipo de estructura societaria; por otro, la necesidad de crear un modelo cooperativo multinacional como principal contribución de la sociedad cooperativa al desarrollo económico del futuro.

Palabras clave: cooperativa agroalimentaria, internacionalización, estrategia, COVAP.

Recibido: 08-10-10. Aceptado: 22-01-11

* Debemos dejar constancia de nuestro agradecimiento por la ayuda recibida de la Agencia Andaluza de Promoción Exterior-EXTENDA y al Director de Internacionalización de COVAP D. Abel Rodríguez.

** Profesora del Área de Organización de Empresas en la Universidad de Córdoba (España). Doctora en CC. Empresariales. Colaboradora de la Cátedra Extenda de Promoción Exterior en la Universidad de Córdoba. e-mail: sandra.sanchez@uco.es.

*** Profesor Titular del Área de Organización de Empresas de la Universidad de Córdoba (España). Doctor en CC. Empresariales y Director de la Cátedra Extenda de Promoción Exterior en la Universidad de Córdoba. e-mail: fernando.fuentes@uco.es.

**** Profesora del Área de Organización de Empresas de la Universidad de Córdoba (España). Licenciada en Administración de Empresas y Derecho. e-mail: td1sarol@uco.es. 


\title{
The Internationalization of Agribusiness Cooperatives. Model of the Spanish Organization, Covap S.C.A.
}

\begin{abstract}
This paper analyzes the situation of cooperative organizations facing the internationalization process, focusing the study on the specific case of agricultural cooperatives, due to their leading role in the economic framework of the European Union. The case analysis method is applied to review the status of the Cooperativa Agraria Valle de los Pedroches (COVAP SCA) (Agrarian Cooperative of the Los Pedroches Valley), located in Andalusia (Spain). This case is presented as an example of how, after 50 years, an originally small cattlemen's cooperative has become one of the first-class agricultural cooperatives, leading sales in Spain and exporting its products to over 23 countries, which include demanding markets such as the United States. Findings show, on the one hand, the requirements and challenges that agricultural cooperatives must face in the internationalization process, in addition to the difficulties associated with this type of societal structure; and, on the other hand, the need to create a multinational cooperative model as a principal contribution from cooperative societies to future economic development.
\end{abstract}

Key words: agribusiness cooperative, internationalization, strategy, COVAP.

\section{Introducción}

A lo largo de las últimas décadas el fenómeno de la internacionalización de empresas ha experimentado una notable expansión, como puede apreciarse en el crecimiento de los movimientos comerciales y el incremento de los flujos de exportación.

Los desencadenantes de la salida al exterior de una empresa no responden a una causa única habitualmente. Mientras que en el caso español se ha achacado a su adhesión a la Unión Europea en 1986, lo que originó un incremento sostenido de la inversión extranjera directa de
España, lo cierto es que la expansión internacional de las pymes españolas fue previa a esta fecha.

Aunque no es posible encontrar una teoría completa sobre la internacionalización (Dicken, 1998) ${ }^{1}$ según Guillén (2004) la salida de las organizaciones al exterior se debe en gran parte a la búsqueda de una forma óptima de explotación de activos comerciales intangibles como marcas y tecnología. De este modo, la inversión en el extranjero no obedece únicamente a razones defensivas, esto es, la respuesta a la llegada de las empresas multinacionales al mercado local, sino que igualmente presenta ca- 
racterísticas ofensivas, similares a las seguidas por las grandes empresas de cualquier país desarrollado.

Tomando como punto de partida a Johanson y Wiedersheim-Paul (1975), para quienes la internacionalización es un proceso secuencial de etapas, el paso de una a otra implica una mayor involucración en los países extranjeros y mayor conocimiento. De esta definición se recoge la idea de que la internacionalización es un proceso de etapas graduales.

Piercy (1981) y Turnbull (1985) describen la internacionalización como el movimiento de expansión de las operaciones de una empresa. Ciertamente, la internacionalización, en la mayoría de los casos (aunque no siempre) se entiende como "un segundo paso", como un mercado adicional cuando el mercado doméstico ya es dominado o llega el momento en que resulta pequeño, es decir, que la expansión en los mercados exteriores depende del crecimiento en el mercado interior.

Pero, a la hora de analizar las causas de la internacionalización, es preciso distinguir entre tres tipos de empresa: las grandes organizaciones, las pymes y las sociedades cooperativas. Este artículo se centra en el caso de estas últimas, por su singular peso en la economía de la Unión Europea (UE), con más de 137.000 empresas (España es el segundo país de la UE en número de cooperativas, tras Italia) que dan empleo a más de 4 millones y medio de personas. Por otro lado, si se analizan los sectores más representativos es incuestionable el peso que suponen las cooperativas agrarias, que alcanzan alrededor del $33 \%$ del total de cooperativas en Europa. En España, por ejemplo, más de dos tercios de los ingresos económicos derivados de sociedades cooperativas provienen de cooperativas agrícolas, lo que viene a representar un tercio de la agricultura española.

Por todo ello, en este trabajo se va a efectuar una revisión teórica del proceso de internacionalización de las cooperativas, centrándonos asimismo en el caso de las cooperativas agrarias, para, a continuación, mostrar un ejemplo de cómo la empresa COVAP S.C.A., situada como la quinta cooperativa agraria por facturación en España (Cogeca, 2010) y ubicada en una comarca del sur de España muy deprimida económicamente ${ }^{2}$, ha sido capaz de formar una sólida empresa, gestionada de manera innovadora que en pocos años ha logrado abrir numerosos mercados exteriores con productos cuya comercialización no es sencilla a priori como son los lácteos, el jamón ibérico, entre otros.

\section{Internacionalización de la empresa cooperativa}

Siguiendo a Mills (2002) y de acuerdo a la Alianza Cooperativa Internacional (ICA), una cooperativa es "una asociación autónoma de personas unidas voluntariamente en la consecución 
Internacionalización de cooperativas agrarias. El modelo de la empresa ...

Sánchez C., Sandra M.; Fuentes G., Fernando J. y Santos R., Luna M.

de necesidades y aspiraciones comunes de índole económica, social y cultural a través de una empresa de la que son propietarios de manera conjunta y es controlada democráticamente". Así, mientras que el objetivo prioritario habitual de las sociedades capitalistas es la maximización del valor para los inversores, las cooperativas suelen buscar este máximo valor tanto a nivel societario como a nivel de cada socio-usuario. Las cooperativas configuran un modelo de empresa alternativo, lo que necesariamente ha de repercutir en su desarrollo internacional.

La situación actual de globalización de la economía y la crisis del sistema capitalista de los últimos dos años ha reabierto la incógnita acerca de la viabilidad del funcionamiento de las cooperativas en este contexto. El debate radica en el análisis de la capacidad que tienen las cooperativas para generar conocimiento e innovación y trascender los límites de su mercado nacional para actuar conjuntamente con otras cooperativas compitiendo con otras empresas capitalistas mientras mantienen simultáneamente sus principios y valores (Errasti et al., 2002). Así, encontramos teorías como la degeneración cooperativa, que predice el fracaso a largo plazo de las cooperativas como organizaciones democráticas debido a las presiones externas del mercado. Sin embargo, esta teoría es rebatida por autores como Cornfoth et al. (1988) que reconocen la posible influencia de estas presiones, pero no su determinismo en el grado en que el control democrático puede ser mantenido por la cooperativa.

Por tanto, la sociedad cooperativa tiene que, en un momento dado, tomar una decisión respecto a la necesidad o no de internacionalizarse, optando entre dos posibles estrategias: especializarse en su mercado manteniendo las ventajas de dicha especialización frente a la competencia, o bien optar por la internacionalización y superar los límites de su mercado local para intentar competir en otras regiones del mundo.

Lo cierto es que, pese a que la mayor parte de cooperativas llevan a cabo su actividad en un ámbito local, no todas logran sobrevivir mediante esta estrategia, ya que los mercados cuentan cada vez con menor protección y las multinacionales pueden entrar a competir con una oferta diferenciada en condiciones más ventajosas. Por ello, también se cuenta con múltiples ejemplos de grandes cooperativas que llevan cierto tiempo inmersas en un proceso de expansión internacional; su desarrollo tanto horizontal como vertical se ha producido por razones muy similares a las de las pymes: intento de penetración en nuevos mercados basado en patentes y tecnología (expansión horizontal) y búsqueda de la reducción de costes de producción, de suministro de inputs y canales de producción para productos nacionales (expansión vertical). Lo que parece innegable es que si se quiere garantizar la supervivencia a largo plazo es necesario competir a nivel mundial (Errasti et al., 2002). Así, Novkovic (2007) afirma que la presencia de las cooperativas en el ámbito internacional se debe principalmente a las presiones competitivas del mercado.

Sin embargo, pese a que se están desarrollando cada vez más los estudios sobre el tema de la internacionalización de las cooperativas (Normark, 1996; Clamp, 2000; Spear, 2001; Errasti et al., 
2003) no existe un marco teórico definido que analice las formas de adaptación de éstas al escenario económico mundial o que haya profundizado en los modos de organización para llevar a cabo el proceso de expansión.

Una conclusión habitual de las investigaciones centradas en este ámbito es que la sociedad cooperativa cuenta con los conocimientos necesarios para resolver la tarea de mantener su estructura democrática en el ámbito de las actividades internacionales. De hecho, Pestoff (1999) considera la dimensión social de las cooperativas como un elemento que las distingue de sus competidores y genera una ventaja competitiva. Así, principios como la autoayuda, la auto-responsabilidad, la igualdad, la equidad y la solidaridad, la tradición de los fundadores, los socios cooperativos y los valores éticos de honestidad, transparencia y vocación social ${ }^{3}$ otorgan a las cooperativas una dimensión superior a la meramente económica. Así se manifiesta Lawler (Aranzadi, 1999), en lo que denomina "ventaja definitiva", que radica en la profunda implicación de las personas en la organización. A su vez, estos principios se vinculan a la importancia de la cooperación entre cooperativas (principio de intercooperación), que puede enlazar con la idea de redes empresariales o "grupos cooperativos" y, en última instancia, generar un gran potencial en el ámbito de la internacionalización. Gómez López (2008) se manifiesta muy favorable a la herramienta de la cooperación entre coo- perativas ya que considera que permite traspasar las estrictas fronteras nacionales para explotar la posibilidad de entablar relaciones con otras sociedades cooperativas homólogas.

Sin embargo, otros autores mencionan la posibilidad de que este principio de intercooperación pueda igualmente generar un efecto barrera a la internacionalización (Davis y Burt, 2007) en tanto el crecimiento de las cooperativas o la fusión entre ellas, puede originar dificultades en la relaciones entre los socios y crear presiones en sus sistemas democráticos y los costes de estructura.

Para Singerman (1987), en cambio, el problema de muchas cooperativas no se refiere tanto a sus objetivos sociales como a la disposición de una adecuada estructura de capital, un buen control financiero y una estrategia de mercado enfocada al exterior. Middleton (1998) argumenta que algunas de las dificultades en la internacionalización de las cooperativas subyacen en las decisiones tomadas por directivos y managers en cuanto al momento y el lugar donde desplegarse internacionalmente y el nivel de compromiso de la cooperativa para permanecer independiente.

En el mismo sentido se manifiestan Donoso et al. (2004) cuando señalan que el futuro de las cooperativas depende en gran medida de la capacidad de sus gestores para crear estructuras competitivas que ofrezcan productos diversificados $y$ de alto valor en un mercado global. Kurimoto (1999) por su parte, considera tam- 
Internacionalización de cooperativas agrarias. El modelo de la empresa ...

Sánchez C., Sandra M.; Fuentes G., Fernando J. y Santos R., Luna M.

bién urgente la necesidad de actualización de las cooperativas respecto a acuerdos de colaboración regional y global en campos como la transferencia tecnológica, aprovisionamientos conjuntos, el intercambio de know-how, o la creación de joint ventures.

Con independencia de estas motivaciones u obstáculos con los que la cooperativa hace frente a su futura internacionalización, es evidente que si ésta desea sobrevivir al proceso no puede convertirse en una multinacional al uso (Errasti, 2004) y en los últimos años es posible comprobar la existencia de cooperativas inmersas en movimientos empresariales multinacionales con un núcleo donde permanece su naturaleza cooperativa y una periferia formada por diversos centros productivos en los que se lleva a cabo un sistema de relaciones capitalistas. El ejemplo más evidente de este caso es la Corporación Cooperativa Mondragón, empresa ampliamente analizada en la literatura especializada (Clamp, 2000; Errasti et al., 2002; Bakaioka et al., 2004; Davies y Burt, 2007).

Asimismo, existen otros casos de intentos de internacionalización en cooperativas (no siempre exitosos). En general, pueden distinguirse dos tipos de proceso de expansión: multinacional e internacional.
- La cooperativa multinacional o cooperativismo articulado puede definirse como una combinación o federación de cooperativas (cada una de ellas establecida inicialmente en un país) que se unen para distribuir productos $y$ servicios a nivel internacional (Craig, 1976). Egerstrom et al. (1996) sugieren que este tipo de multinacional puede surgir de la fusión de dos o más cooperativas o bien, de la creación de una forma de holding cuyos miembros son cooperativas regionales o nacionales; el holding adquiriría la forma de sociedad capitalista mientras que las partes permanecen con la estructura institucional de cooperativas. No obstante, esta forma de inter-cooperación internacional se enfrenta a problemas como: divergencias en las regulaciones jurídicas entre países en lo que al concepto "cooperativa" se refiere ${ }^{4}$; limitación en el número de cooperativas en ciertos sectores industriales a la hora de buscar socios internacionales; dificultad de lograr acuerdos entre comunidades de trabajadores-socios con intereses muy diversos.

- Cooperativas internacionales. Se trata del modelo alternativo en que la expansión en un mercado internacional se lleva a cabo por una única cooperativa. En general esta situación se origi-

No obstante, la creación de estructuras asociativas de mayor tamaño y con ámbito territorial transfronterizo o transnacional ha sido apoyado desde las instituciones oficiales europeas y como claro ejemplo de ello se aprueba en el año 2003 desde el Consejo Europeo el Estatuto de la Sociedad Cooperativa Europea (SCE), Reglamento (CE) n 1435/2003 del Consejo del 22 de julio de 2003. Este estatuto proporciona a las cooperativas los instrumentos necesarios para el desarrollo de sus actividades en el ámbito internacional. 
na cuando se efectúa una estrategia ofensiva de marketing para añadir valor a los productos de la cooperativa. El propósito es conseguir el mejor precio para generar un beneficio extra para los socios y la comunidad local. Ello puede significar simplemente la venta a nivel internacional (caso de Covap S.C.A) o la inversión de capital en el extranjero para fortalecer la producción y las actividades comerciales y mediante la constitución de filiales en el extranjero. Algunos autores como Errasti et al. (2002), no obstante, consideran que la esencia del cooperativismo, basada en la soberanía del trabajo (un socio-un voto) puede quedar diluida en este marco de creación de filiales en el extranjero configuradas por trabajadores asalariados por cuenta ajena. Hay que añadir, por otro lado, que numerosas tentativas de internacionalización de cooperativas han fracasado y aquellas que permanecen, todavía facturan en muchos casos a una escala reducida.

En cuanto al impacto del proceso de internacionalización en los resultados de la cooperativa, los estudios empíricos sugieren una relación directa (Sullivan, 1994) aunque, según Geringer et al. (1989), cuando se alcanza cierto grado de internacionalización, operar en un mayor número de mercados conduce a costes más altos derivados de las distancias geográficas y esto acaba por reducir los márgenes de beneficio.

En opinión de autores como Bakaikoa et al. (2004) el éxito de la empresa cooperativa es una cuestión de cierta complejidad donde entran en juego variables como la creación de empleo y riqueza, la aportación a la economía del entorno, la relaciones con la comunidad, la cuestión ecológica y, en lo referente a las relaciones internacionales, la capacidad de transformar las relaciones económicas en términos de "cooperación y democracia" como principios clave de funcionamiento de este tipo de sociedades.

\subsection{Sector agroalimentario: Política Agraria Común (PAC) y retos futuros}

El sector agroalimentario tanto en España como en la Unión Europea según Bocherini (2010) se caracteriza por una madurez e intensidad competitiva debido a diversos factores (un consumo que crece poco, competencia empresarial, reducción de los mecanismos de intervención y protección estatal, liberalización progresiva del comercio internacional con entrada de productores que compiten con menores costes y más liberalizados).

Por su parte, Baamonde (2009) destaca que desde 1992 la Política Agraria Común (PAC) de la Unión Europea ha ido desmantelando progresivamente sus mecanismos de intervención, pretendiendo que la operativa del mercado «pusiese a cada uno en su sitio». Así transformó los instrumentos de garantía de precios en ayudas directas desacopladas, olvidándose de los principios fundamentales que inspiraron la PAC en el momento de su creación. Estos principios no son otros que la garantía de rentas y de abastecimiento, precios razonables al consumo y la preferencia comunitaria, principios que, en la práctica actual, ya no están vigentes.

La eliminación de excedentes ha sido la obsesión permanente de la Comi- 
Internacionalización de cooperativas agrarias. El modelo de la empresa ...

Sánchez C., Sandra M.; Fuentes G., Fernando J. y Santos R., Luna M.

sión europea y su eliminación el objetivo prioritario. Pero en los últimos años, el crecimiento de la demanda internacional, sobre todo en los países emergentes, unido a la aparición de nuevos usos no alimentarios para la obtención de biocarburantes y a la reducción de la producción en los principales países exportadores como consecuencia de la climatología adversa, han hecho que los precios de las materias primas (cereales, oleaginosas, leche y productos lácteos, entre otros) hayan experimentado un incremento extraordinario y se llegue a graves problemas de volatilidad de precios (Baamonde 2009).

Hasta el momento, la PAC se ha asentado en dos pilares básicos financiados con un $1 \%$ del gasto público de la UE: por un lado, beneficios por el total de la actividad agrícola instrumentados en gestión del mercado y pagos directos a los agricultores; por otro, beneficios proporcionados por los agricultores en su papel de gestores de tierras, de forma voluntaria a cambio de pagos específicos, garantizados por la sección de Desarrollo Rural de la PAC. De cara al futuro, existe una serie de circunstancias que hacen peligrar estos beneficios, especialmente los referentes al primer pilar: aumento de inestabilidad en los mercados, condiciones desiguales en los agricultores para afrontar las importaciones; rentas de los agricultores deterioradas aún más por la crisis económica; retos derivados del cambio climático. Por todo ello, COPACOGECA (2010) recoge una serie de objetivos y principios orientadores de la futura PAC a partir de 2013 entre los que destaca "proporcionar un marco estable para el desarrollo de la producción agrí- cola, a través de un aumento de la productividad y de la competitividad, así como para el adecuado funcionamiento del mercado"

Parece inevitable que, en este contexto, las empresas cooperativas agroalimentarias tengan la exigencia de salir a nuevos mercados como forma de respuesta a esta situación.

\subsection{Cooperativas agrarias $e$ internacionalización}

Como se acaba de precisar el sector agroalimentario se enfrenta a un entorno competitivo complejo y difícil, en el que el bajo crecimiento del consumo y la abundancia de oferta presionan constantemente los precios a la baja. Tradicionalmente la forma más habitual de combatir la atomización empresarial en el sector primario ha sido la creación de cooperativas que aglutinan socios que voluntaria y democráticamente acuerdan objetivos. En este sector generalmente se ocupan de almacenar, transformar, comercializar $y$, en definitiva, permitir que pequeños productores incrementen su poder de negociación y alcancen economías de escala y de alcance a las que individualmente no tendrían acceso.

El estudio del proceso de internacionalización en las cooperativas agrarias es de particular interés principalmente por la falta de investigaciones en este campo (Donoso et al., 2003) y la creciente importancia de este sector.

Las cooperativas agrarias han desarrollado gradualmente en distintos países una estrategia de diversificación, como alternativa de entrada a nuevos mercados para comercializar sus produc- 
tos en el extranjero y levantar barreras proteccionistas (Ritossa y Bulgacov, 2009). La internacionalización se ha llevado a cabo mediante dichas acciones de diversificación en los productos ofertados en los mercados exteriores.

Aunque este tipo de cooperativa se creó para ofrecer apoyo a las familias de los productores agrícolas, los cambios internacionales han influido en los mercados donde operan estas sociedades. Se hace patente la necesidad de equilibrar los intereses de los socios con la dinámica competitiva del entorno. La opinión de los miembros de la cooperativa acerca de la estrategia de internacionalización va a depender, como es lógico, de los resultados que se deriven de las operaciones realizadas en los mercados exteriores.

Las razones que motivan el proceso de internacionalización de una cooperativa agraria son similares a las de otras formas de organización (FCD, 2000) aunque Seipel y Hefferman (1997) añaden algunas ventajas de este tipo de cooperativa que redundan en la línea de la necesidad de abrirse a nuevos mercados extranjeros: por un lado, los socios agricultores o ganaderos garantizan la alta calidad de los productos que suministran, lo que incrementa la percepción de seguridad e higiene alimentaria en el exterior; por otra parte, las cooperativas suelen percibirse como posibles socios comerciales éticos y fiables.

Ritossa y Bulgacov (2009), en su estudio sobre cooperativas agrarias de Brasil confirman que las más favorables a la idea de la internacionalización presentan características tales como certificaciones de seguridad alimentaria, nivel tecnológico similar al de los mercados de- sarrollados o acceso a canales de distribución extranjeros. Las principales barreras percibidas por las cooperativas de esta investigación se centran en factores externos (burocracia y asuntos legales relacionados con el proceso de internacionalización, costes de cambio o costes de transporte superiores originados por una pobre estructura logística).

En el caso de las cooperativas agrarias españolas (que suponen más del $70 \%$ del total de cooperativas del país) las estadísticas muestran un sector donde predominan las sociedades de primer grado, un $86 \%$ según el Observatorio Socioeconómico del Cooperativismo Agrario Español (OSCAE), (referenciado en Cooperativas Agroalimentarias, 2010). La base exportadora es relevante (entre un 31 y un $44 \%$ de las cooperativas de este sector de primer y segundo grado respectivamente exportan habitualmente, según la Confederación de Cooperativas Agrarias de España CCAE). El volumen de exportación de éstas supone alrededor de un $15 \%$ del total de exportaciones agroalimentarias españolas y se está iniciando una tendencia a ampliar los mercados de destino desde los tradicionales países de la Unión Europea (Alemania, Francia, Portugal, Reino Unido y Holanda principalmente) a otros grandes mercados no europeos (Estados Unidos, Japón o Canadá) e incluso nuevos mercados emergentes (China o India).

Además, las cooperativas agrarias españolas presentan una capacidad adecuada para organizar y ofertar grandes volúmenes de distintas gamas de productos con la garantía de una eficaz trazabilidad, proximidad al productor y óptimas condiciones de seguridad alimentaria, 
Internacionalización de cooperativas agrarias. El modelo de la empresa ...

Sánchez C., Sandra M.; Fuentes G., Fernando J. y Santos R., Luna M.

respeto al medioambiente y al bienestar de las ganaderías.

En cuanto a las estrategias de internacionalización, mayoritariamente (un $66,8 \%$ según el estudio de la CCAE (2010) se decantan exclusivamente por las exportaciones Martín (2006) y Fernández et al. (2008) resaltan que las exportaciones contribuyen a mejorar la utilización de la capacidad productiva de la empresa, a desarrollar capacidades de gestión superiores, a mejorar la calidad de procesos e innovar y a fortalecer resultados. Sólo el 7,3\% buscan la cooperación internacional con otras cooperativas.

Pese a todo ello, la implantación exterior de las cooperativas españolas es escasa, sólo el $7,41 \%$ de las cooperativas de segundo grado se han establecido en el exterior y apenas la mitad en las cooperativas de primer grado. Precisamente Boccherini (2010) en relación a la agroindustria alimentaria española destaca que presenta un escaso interés por la internacionalización. La implantación en el exterior es ciertamente una tarea compleja, no exenta de riesgos y dificultades y muy consumidora de recursos directivos (tan escasos en las cooperativas). De hecho, la baja internacionalización se debe, en muchos casos, a los problemas de dimensión y formación empresarial.

Por ello, en los últimos años parece imparable la tendencia a la creación de cooperativas de segundo grado lo que supone un incremento del poder de negociación en los mercados y alcanzar economías de escala inasequibles a las cooperativas más pequeñas.

\subsection{Casos de internacionalización de cooperativas agrarias en la UE}

La creación de cooperativas de mayor tamaño que buscan modelos más cercanos a la estructura de sociedad anónima (Gómez, 2008) ya está ocurriendo con fuerza en países del norte de Europa como Dinamarca, Países Bajos o Suecia donde las cooperativas ocupan los primeros puestos de facturación en el entorno productivo agrícola europeo (Cogeca, 2010). Según Juliá y Martín (2008) los países de la Unión Europea con cooperativas agrarias de mayor tamaño (Dinamarca, Finlandia, Irlanda, Holanda y Suecia) son aquellos en los que dichas cooperativas poseen una mayor cuota de mercado y, de hecho, concluyen que es la estrategia de internacionalización la que las ha hecho líderes en sus respectivos mercados.

Así, en Dinamarca, MD Foods, cooperativa del sector lácteo, presenta una estrategia de internacionalización mediante inversiones directas y participaciones fuera de su mercado local (concretamente en Alemania y Reino Unido). También se ha creado una cooperativa transfronteriza y transnacional con su fusión con la cooperativa sueca Arla. Por su parte, Danish Crown permite la participación de capital ajeno a través de filiales con estructura de sociedad anónima. También en el sector cárnico ha habido fusiones de cooperativas en este país.

En Holanda también se han producido casos de concentración de cooperativas, como el núcleo intercooperativo The Greenery International en el mercado de las frutas y hortalizas, con una am- 
plia red de centros filiales en Holanda, Alemania, Bélgica, España e Italia. De hecho, las cooperativas agrícolas holandesas han desarrollado una estrategia de ampliación sin restricciones donde se ha admitido la afiliación de nuevos socios sin condiciones financieras. Sin embargo, Hendriske y Oijen (2002) concluyen en su estudio cobre cooperativas holandesas que su grado de diversificación es inferior al de las sociedades capitalistas.

También Theuvsen y Ebneth (2005) analizan el proceso y estrategias de internacionalización para el caso de cuatro cooperativas agrarias de Holanda (Campina), Dinamarca (Danish Crown) y Alemania (Nordmilch y Westfleisch). Como principal conclusión destacan el menor grado de internacionalización en las compañías alemanas. Según los autores, esta circunstancia obedece al menor tamaño del mercado local de las cooperativas holandesas y danesas, que les obliga a mejorar su eficiencia dirigiéndose a los mercados internacionales.

Por su parte Davies y Burt (2007) mencionan el caso de Coop Norden, creada como una joint ventue de tres cooperativas minoristas escandinavas, como ejemplo de cooperativa multinacional derivada de una forma de holding entre cooperativas regionales o nacionales como miembros de éste. Igualmente, destacan la Corporación Cooperativa Mondragón (mencionada previamente en este trabajo) como caso de singular crecimiento $y$ estrategia de unión bajo una misma reseña como forma de protección de la cooperativa ante la competencia. Otro caso analizado fuera de Europa es el de NTUC FairPrice de Singapur, uno de los ejem- plos de internacionalización más prolíficos en cooperativas de consumo.

En definitiva, se percibe que tamaño y grado de internacionalización poseen una relación directa en estos países (Juliá y Meliá, 2008) como se aprecia en los casos de Arla Foods (mayor cooperativa sueco-danesa), Friesland Coberco Diary Foods y Campina (primera y segunda cooperativa por tamaño en Holanda), Glambia (segunda cooperativa en tamaño en Irlanda) y el Grupo Valio (segundo en Finlandia).

\section{La internacionalización de la cooperativa del Valle de Los Pedroches (Covap)}

\subsection{Historia de Covap}

El origen de esta Cooperativa se remonta al año 1959 en que un grupo de 10 ganaderos de la localidad de Pozoblanco, en el Valle de Los Pedroches (Córdoba, España) deciden asociarse para la adquisición en mejores condiciones de pago, de una partida de maíz como alimento para su ganado. Esta asociación coyuntural se convertiría en el germen de lo que finalmente se consolidó en cooperativa. El proyecto inicial de la misma sería la construcción de una fábrica de piensos que se lleva a término en el año 1963, ampliándose de ese modo el ámbito de actuación de la Cooperativa y permitiendo su posterior desarrollo. Más adelante los socios comprendieron que era necesario afrontar el problema de la distribución de sus productos perecederos y que debido a la lejanía de Pozoblanco de los circuitos de distribución era con- 
Internacionalización de cooperativas agrarias. El modelo de la empresa ...

Sánchez C., Sandra M.; Fuentes G., Fernando J. y Santos R., Luna M.

veniente que también la Cooperativa se ocupara de la transformación y la comercialización.

Así, los años siguientes son un claro exponente de la continua evolución en la gama de productos y servicios desarrollados por Covap, con la progresiva construcción y modernización de plantas y centros de tipificación. Son ejemplos significativos de este desarrollo la instalación de centros de recogida de leche en 1976, la creación del Matadero e Industria del cerdo ibérico en 1986 o la inauguración de la Sección de Crédito en 1996, entre otros múltiples proyectos.

En la actualidad, las explotaciones de los socios de Covap se ubican en tres áreas limítrofes del Norte de Córdoba (Valle de los Pedroches), Ciudad Real (Valle de la Alcudia) y Badajoz (Valle de la Serena). Se trata de una zona deprimida con grandes llanos de pastizal, mejores para la actividad ganadera en tanto los suelos son poco productivos para la agricultura.

La Cooperativa alcanza en el momento presente los 15.000 socios, lo que supone más del doble de la cifra de cooperativistas con que contaba en 1990 (6.067). La Asamblea General de Delegados elegidos en Juntas Preparatorias es el órgano que representa la voz de los socios en el seno de la Cooperativa. Es esta Asamblea la que nombra al Consejo Rector (incluido el presidente) que ejerce de órgano decisorio. Asimismo, cada una de las actividades tiene una comisión constituida por ganaderos de la misma y que representan a las distintas zonas donde opera la Cooperativa, teniendo como misión elevar distintas propuestas al Consejo Rector para su aprobación.
En 2006 se produjo un intento de transformación de la estructura societaria por las dificultades políticas, estratégicas y financieras para el desarrollo de los negocios que podía presentar una forma de Cooperativa. Se pensó entonces en un cambio en la organización para afrontar con éxito los nuevos retos de mercado mediante la transformación en Sociedad Anónima. Sin embargo, un grupo de socios, que se habían agrupado bajo la plataforma "Todos con Covap", cifrada en torno al $60 \%$ del total de cooperativistas, rechazó el proyecto al entender que suprimiría el "carácter fundacional de la cooperativa" y conllevaría una "quiebra importante" de la economía social en las comarcas en las que opera. Con ello se demuestra la vocación cooperativista de los socios.

En 2009, con motivo del 50 Aniversario de la fundación de la Cooperativa, su Presidente, Ricardo Delgado aboga por la idea de que Covap gane más dimensión empresarial para competir y sobrevivir, insistiéndose en la necesidad de que las cooperativas lleven a cabo alianzas "independientemente del lugar donde unas y otras estén ubicadas, incluso de índole internacional, porque es la única manera de seguir adelante dado la situación de crisis que actualmente estamos viviendo".

En estos 50 años, Covap ha pasado de ser una más de la multitud de cooperativas que surgieron en toda España a convertirse en el motor de desarrollo de la zona norte de la provincia de Córdoba. Puede afirmarse que Covap es hoy la cooperativa más importante de Andalucía, controlando gran parte del mercado andaluz de la leche y la carne y alcanzando 
una cifra de negocio que rondó los 300 millones de euros en 2009.

\subsection{Modelo de negocio de Covap}

Según se aprecia en la figura 1 , Covap ha basado su modelo de negocio en la forma de Sociedad Cooperativa con una preocupación constante por la figura del socio. Sus tres áreas funcionales presentan un alto grado de integración vertical. La sección de suministros se encarga del abastecimiento de piensos, materias primas y mezclas a los socios ganaderos de la organización. Asimismo, desde el área de servicios complementarios se ofrece un integral asesoramiento técnico, así como una sección de crédito, destinados en ambos casos al apoyo permanente al ganadero.

De hecho, Covap está desarrollando una labor de protección para sus socios en la actual crisis económica. Y es que entre los principios que basa su actuación se encuentran: el servicio a los socios, "trabajando para resolver sus problemas y hacer por ellos lo que individualmente no son capaces"; la mejora de sus rentas, abaratando los suministros y transformando y comercializando la producción; la voluntariedad, dado que los socios poseen libertad para asociarse a aquellas actividades que deseen; el pago por calidad, no habiendo diferencias por cantidades o distancias en lo que paga el socio por productos o servicios, pero existiendo un sistema de bonificaciones y penalizaciones en el pago al socio por los productos entregados, lo que se traduce en un estímulo para los cooperativistas y una garantía de calidad para el consumidor.
Por otro lado, las industrias alimentarias Covap, organizadas en función de la cabaña de ganado (vacuno de carne y de leche, caprino, ovino y cerdo ibérico) cuentan con una serie de instalaciones y centros en continuo proceso de crecimiento y sucesivas ampliaciones. El plan de inversiones 2000-2007 ha supuesto un volumen de 150 millones de euros y se ha traducido en el levantamiento de la planta láctea más moderna de Europa, un nuevo Centro de Tipificación de Corderos, un centro de cría de lechones, la ampliación del Centro de Tipificación de Terneros, un nuevo matadero, la ampliación de la industria del cerdo ibérico, una nueva planta de piensos y la puesta en marcha de una planta de platos preparados.

Este sistema altamente integrado verticalmente, permite a Covap realizar una apuesta decidida por la calidad, lo que ha trasladado de manera constante a sus métodos de trabajo $\mathrm{y}$, de hecho, forma parte de su concepto de marca "Calidad desde el origen". El estricto control sanitario y alimenticio al que la cooperativa somete todos sus productos ha permitido que entre los logros de Covap destaque el hecho de haberse convertido en la primera empresa andaluza en obtener el certificado de calidad AENOR ISO 9002 para los productos lácteos. La fábrica de piensos y Centro de Tipificación de Terneros se encuentran acreditados por BVQI. Además, este centro, junto con el de Corderos, dispone de un sistema de certificación de productos cárnicos expedido por CERTICAR. Por su parte, la Planta de Piensos ha sido certificada por CESFAC. Asimismo, la empresa perte- 
Internacionalización de cooperativas agrarias. El modelo de la empresa ...

Sánchez C., Sandra M.; Fuentes G., Fernando J. y Santos R., Luna M.

nece a la Asociación de Usuarios del Distintivo de Calidad Alimentos de Andalucía.

Entre sus objetivos estratégicos prioritarios Covap pretende llegar al cliente a través del mayor número de canales disponibles. De este modo, aparte de las redes de distribución habituales (cadenas de hipermercados y supermercados, mayoristas, hostelería y restauración, cadenas de charcuterías y carnicerías) la Cooperativa ha desarrollado una red de tiendas de calidad especializadas donde el consumidor puede adquirir directamente el producto Covap (diagrama 1).

Con todo ello, el constante crecimiento en la facturación (gráfico 1) logrado por un sucesivo incremento de la cuota de mercado nacional, unido a la política financiera de reinversión de beneficios en un incremento permanente de activos, conforman las bases de la fortaleza de la cooperativa, según el director general, Miguel Angel Díaz Yubero. Para éste, las recientes cifras de ventas convierten a Covap en la cooperativa española de primer grado líder de facturación dentro del sector agroalimentario, y la quinta si se extiende el arco a las cooperativas de segundo grado.

Estas cifras y el deseo de apostar por la apertura internacional llevaron a la Cooperativa a aventurarse en el mercado extranjero con la implantación en el año 2000 del Departamento Internacional. El proceso de internacionalización se analizará en más detalle en el siguiente epígrafe.

La estructura financiera de la Cooperativa apuesta por un equilibrio entre las partidas corrientes y no corrientes (gráfico 2), con un fondo de maniobra suficiente. Los recursos propios han pasado de una cifra de 12,2 a 92 millones de euros en los últimos veinte años. Unas cuentas permanentemente saneadas y un respaldo decidido por parte de distintas entidades y organismos públicos (en el caso del proceso de internacionalización cabe destacar el apoyo de Extenda o el ICEX) permiten a Covap gozar de una fortaleza económica con la que afrontar posibles escenarios de inseguridad financiera (tal y como sucede en el momento de crisis presente).

Finalmente, pero no por ello menos importante, el Capital Humano con el que cuenta la Cooperativa se encuentra ampliamente formado y comprometido con la idea de calidad, gracias a un elevado porcentaje de estabilidad en el empleo. Este aspecto unido a todo lo anterior hacen que COVAP posea ya una andadura de 50 años plagados de logros, marque un referente en el sector agroalimentario español y, cada vez más, en el mercado internacional (cuadro 1, gráfico 3).

\subsection{La Internacionalización de Covap}

Tal y como se mencionaba en el análisis del modelo de negocio, es en el año 2000 cuando se produce el inicio del proceso de internacionalización de Covap con la creación del Departamento Internacional. Son dos los principales objetivos que se marca desde el inicio de su andadura: por un lado, iniciar la trayectoria exportadora de la cooperativa, y en segundo lugar, alcanzar acuerdos de colaboración con empresas extranjeras para la transferencia de tecnología y know-how. 


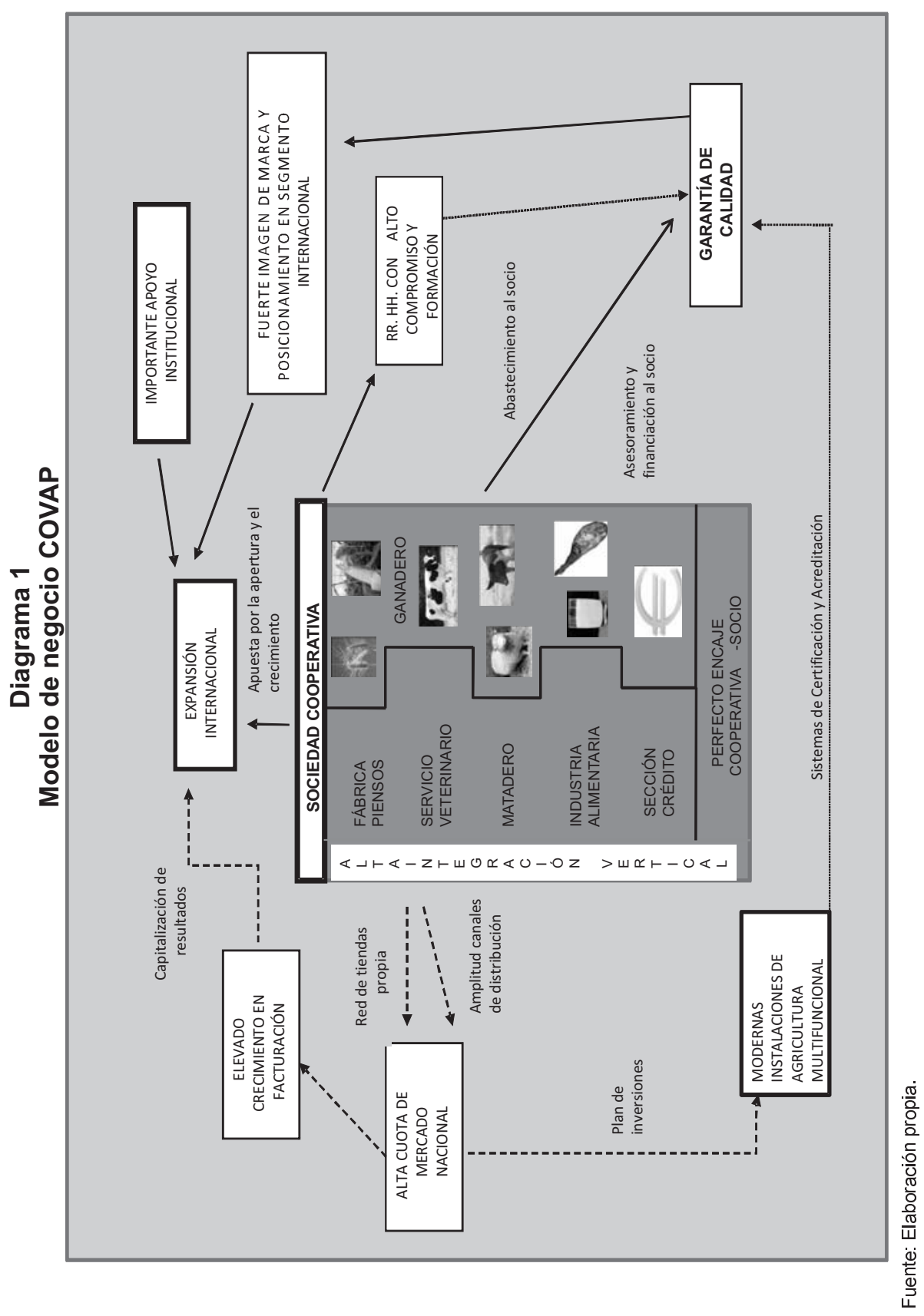




\section{Gráfico 1 \\ Evolución ventas COVAP 1998-2009 (Millones de Euros)}

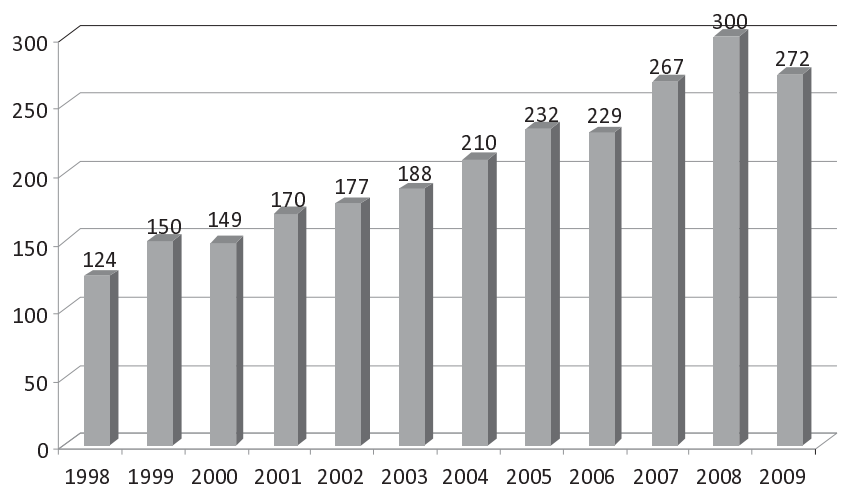

Fuente: Elaboración propia con base en los informes anuales COVAP.

Gráfico 2

\section{Estructura de balance Covap}
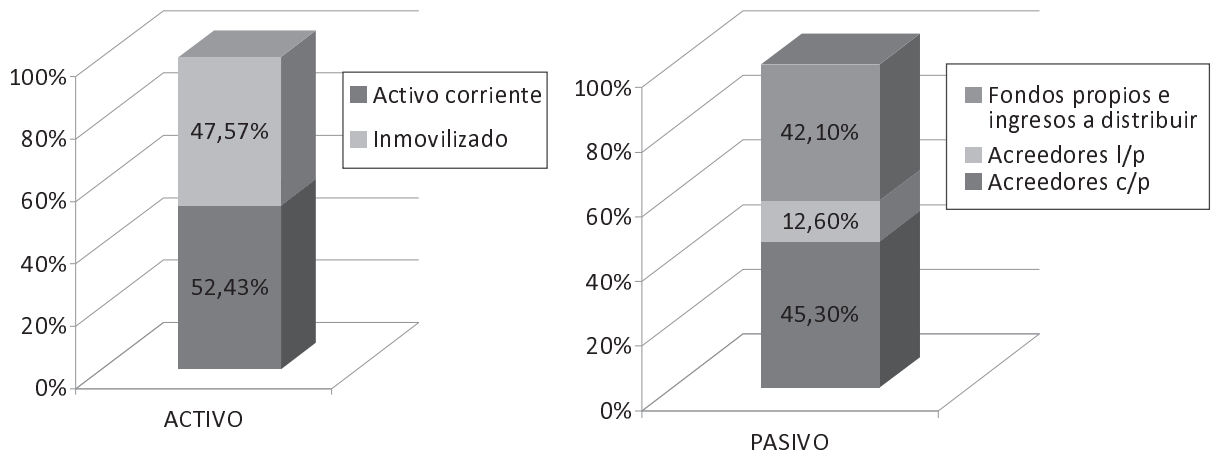

Fuente: Elaboración propia con base en las Cuentas Anuales Covap.

Según el Director del Departamento Internacional, Abel Rodríguez, puede afirmarse que en el período 2000-2009 los objetivos alcanzados han sido los siguientes:

- Apertura de nuevos mercados: presencia constante en 23 países en el 2008.

- Creación de la Marca COVAP, como referente de calidad agroalimentaria.
- Introducción en los canales apropiados de cada producto en los mercados exteriores.

- Acuerdos de colaboración con empresas/gobiernos extranjeros para la transferencia de tecnología y knowhow.

La estructura organizativa de este departamento quedaría formada por dos áreas manager dependientes del Direc- 


\section{Cuadro 1 \\ Elecciones clave en el modelo de negocio COVAP}

\begin{tabular}{|c|c|}
\hline Decisiones & Descripción \\
\hline Diseño & $\begin{array}{l}\text { - Moderna agricultura multifuncional y respetuosa con el me- } \\
\text { dioambiente. } \\
\text { - Protagonismo de los ganaderos }\end{array}$ \\
\hline Gama & $\begin{array}{l}\text { - Amplio surtido de lácteos, cárnicos y platos preparados. } \\
\text { - Suministro de piensos, mezclas y mercaderías al socio que ga- } \\
\text { rantiza la calidad final del producto }\end{array}$ \\
\hline Marketing & $\begin{array}{l}\text { - Presencia en ferias y stands gastronómicos internacionales } \\
\text { - Posicionamiento de marca en el segmento alto del mercado in- } \\
\text { ternacional }\end{array}$ \\
\hline Concepto & $\begin{array}{l}\text { - Creación de marca COVAP como referente de calidad agroali- } \\
\text { mentaria }\end{array}$ \\
\hline Producción & $\begin{array}{l}\text { - Modernas instalaciones bajo estricto control sanitario y alimenti- } \\
\text { cio } \\
\text { - Automatización de procesos en un sistema integrado vertical- } \\
\text { mente }\end{array}$ \\
\hline Expansión internacional & $\begin{array}{l}\text { - Presencia en } 23 \text { países y acuerdos de colaboración con empre- } \\
\text { sas/gobiernos extranjeros para la transferencia de tecnología y } \\
\text { know-how }\end{array}$ \\
\hline Personal y RR.HH. & $\begin{array}{l}\text { - Más de } 600 \text { empleados en la actualidad, lo que supone una cifra } \\
5 \text { veces superior en una década (gráfico 4). } \\
\text { - Estabilidad de empleo superior al } 90 \% \text { y edad media de la plan- } \\
\text { tilla de } 36 \text { años. }\end{array}$ \\
\hline Ventajas competitivas & $\begin{array}{l}\text { - Diferenciación mediante garantía de calidad del producto desde } \\
\text { su origen } \\
\text { - Sistemas de seguimiento y trazabilidad más avanzados del mer- } \\
\text { cado }\end{array}$ \\
\hline Estructura financiera & $\begin{array}{l}\text { - Reinversión de beneficios que ha permitido un sucesivo incre- } \\
\text { mento de activos. } \\
\text { - Equilibrio en las partidas de pasivo en una estructura financiera } \\
\text { firmemente asentada y saneada en su composición (gráfico 3). }\end{array}$ \\
\hline Estructura organizativa & $\begin{array}{l}\text { - Altamente participativa. Integración de los socios en la gestión } \\
\text { organizativa } \\
\text { - Comisiones de ganaderos por zonas que elevan propuestas al } \\
\text { Consejo Rector. }\end{array}$ \\
\hline
\end{tabular}

Fuente: Elaboración propia. 
Internacionalización de cooperativas agrarias. El modelo de la empresa ... Sánchez C., Sandra M.; Fuentes G., Fernando J. y Santos R., Luna M.

tor. Por un lado, el área Europa del Este, Asia y Oceanía (desde abril de 2008) y por otro, el área América y África, aún pendiente de implantar. Existe asimismo un asistente para todas las áreas del que depende jerárquicamente un administrativo.

En cuanto a la evolución de las exportaciones, se puede afirmar que en menos de una década ha experimentado un auge importante gracias a la constante expansión de la cooperativa en distintos mercados internacionales. Tal y como se observa en el gráfico 4 , el ritmo de crecimiento ha sido constante y especialmente significativo desde su inicio, salvo para el ejercicio 2007, donde se produjo un descenso del $23 \%$ en la facturación a causa de la apreciación del euro frente al dólar, el aumento en los precios de vacu-

Gráfico 3

\section{Evolución de la cifra de empleados COVAP}

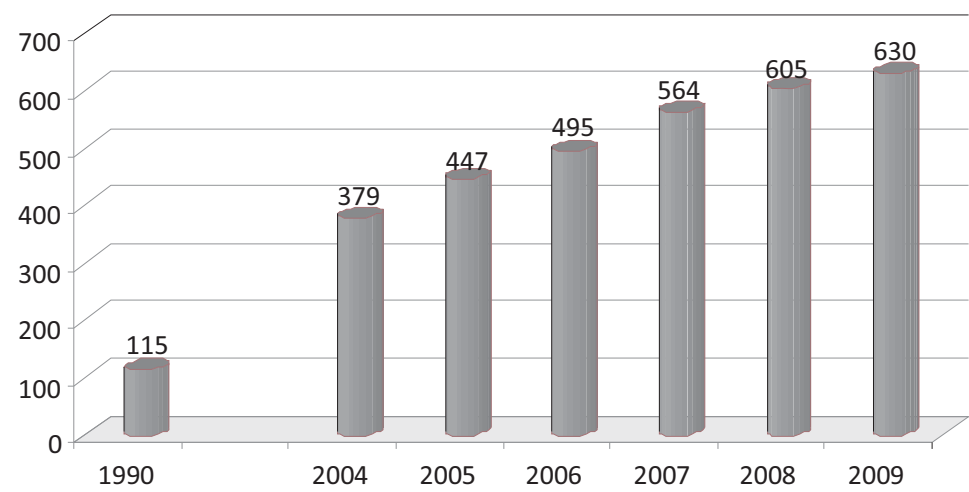

Fuente: Elaboración propia con base en los informes anuales COVAP.

\section{Gráfico 4 \\ Evolución exportaciones 2001-2009 (euros)}

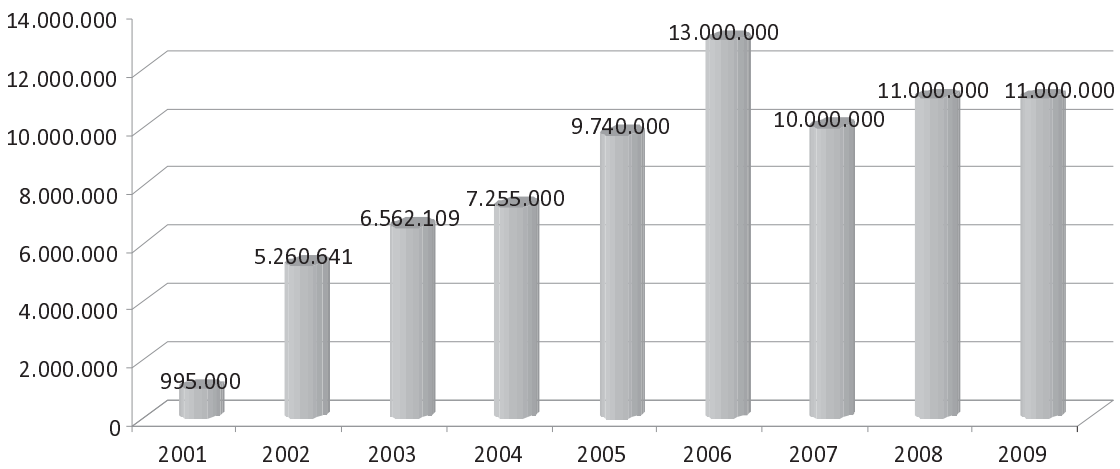

Fuente: Elaboración propia con base en los informes anuales COVAP. 
no y ovino por la subida del precio del cereal y la disminución en la capacidad de suministros de nata a granel.

Los principales porcentajes de exportación se corresponden con los productos de ovino y vacuno, seguidos de los lácteos e ibéricos. En cuanto a los países de destino de las exportaciones en cada grupo, el cuadro 2 muestra cómo la Unión Europea es el destino preferente en todos los productos, especialmente en el caso de los ibéricos, que tienen presencia en 14 países del continente, así como en otros 6 países asiáticos y americanos. Los quesos forman otro grupo con amplia representación internacional. Igualmente, hay que destacar el nuevo grupo de platos preparados que se está abriendo camino en los mercados francés e italiano así como en Hong Kong.

En lo referente a volúmenes de facturación por países destacan en gran me- dida Italia y Portugal con exportaciones de 5,5 y 2,5 millones de euros respectivamente. Por otra parte, hay que subrayar la presencia de Japón, Hong Kong y Estados Unidos dentro de las diez primeras posiciones. En la actualidad, COVAP cuenta con 71 clientes extranjeros distribuidos en 23 países diferentes (gráfico 5).

\subsection{Retos de futuro}

Pese a la situación económica actual, Covap obtuvo en 2008 un crecimiento del $21 \%$ para las ventas de leche, columna vertebral de la Cooperativa. Sin embargo, el sector de ibérico sufrió una caída de ventas del $10 \%$ arrastrado por el exceso de producción que la demanda no pudo absorber, generando además tensiones en los precios. En 2009, pese a la caída de precios, incrementando las can-

\section{Cuadro 2}

Destinos de la exportación de productos COVAP

\begin{tabular}{|c|c|c|c|}
\hline Producto & Europa & Asia & América \\
\hline Ibéricos & $\begin{array}{l}\text { Alemania, Austria, Bélgica, } \\
\text { Chequia, Francia, Grecia, Holanda, } \\
\text { Irlanda, Italia, Luxemburgo, } \\
\text { Noruega, Suecia, Portugal, Reino } \\
\text { Unido }\end{array}$ & $\begin{array}{l}\text { China, Hong Kong, } \\
\text { Japón }\end{array}$ & $\begin{array}{l}\text { Chile, Martinica, } \\
\text { México y EEUU }\end{array}$ \\
\hline Quesos & $\begin{array}{l}\text { Alemania, Chequia, Francia, } \\
\text { Holanda, Irlanda, Portugal, Rusia }\end{array}$ & China, Hong Kong & $\begin{array}{l}\text { Chile, EE.UU., } \\
\text { Martinica, México }\end{array}$ \\
\hline Ovino y Vacuno & $\begin{array}{l}\text { Alemania, Francia, Holanda, Italia, } \\
\text { Portugal }\end{array}$ & & \\
\hline Lácteos & $\begin{array}{l}\text { Francia, Grecia, Portugal, Reino } \\
\text { Unido }\end{array}$ & & \\
\hline Platos & Francia, Italia & Hong Kong & \\
\hline Lana Merina & Reino Unido & & \\
\hline
\end{tabular}

Fuente: Elaboración propia con base en los informes anuales COVAP. 


\section{Gráfico 5 \\ Principales países por porcentaje de exportación (10 primeras posiciones)}
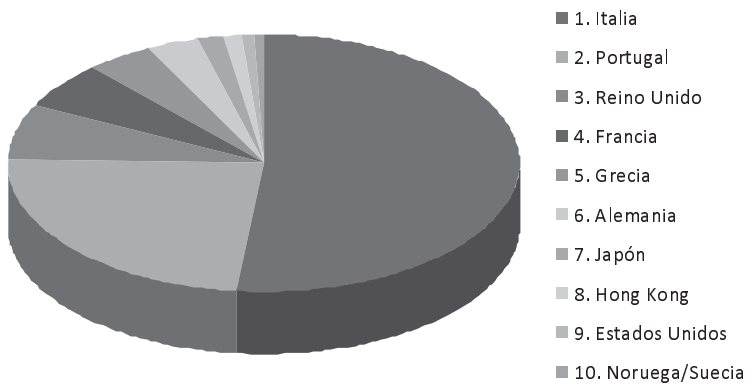

Fuente: Elaboración propia con base en los informes anuales COVAP

tidades de producto vendidas se consiguió mantener la cifra de ventas.

Ante el escenario económico actual, Covap se plantea una serie de actuaciones a corto y medio plazo. Por un lado, se pretende plantear la modificación de los estatutos sociales de la entidad, que afectarán a cuestiones como los socios inactivos, la composición del consejo rector, las aportaciones a capital Social, o la distribución de los excedentes. Paralelamente, se ha anunciado la elaboración antes de un plan estratégico para el periodo 2010-13 cuyas líneas están por definir.

Por otro lado, Covap se ha incorporado recientemente como socio a la cooperativa de segundo grado Cordesur, constituida por Corsevilla y Dehesas Cordobesas (Corpedroches), como una plataforma donde se concentre la oferta con el ánimo de regular el mercado del ovino andaluz. También se está estudiando el planteamiento de alianzas con grandes cooperativas de alimentación andaluzas como Hojiblanca o Agrosevilla, pretenden incidir en la línea de abaratar costes y adquirir dimensión, que, en particular per- mitirán reforzar la presencia en mercados exteriores.

En 2010 COVAP ha conseguido las autorizaciones que permitirán la exportación de sus productos de cerdo ibérico a Estados Unidos, un permiso que sólo tenía anteriormente otro matadero español. Los productos se irán introduciendo de forma escalonada ya que sólo se podrán exportar los ejemplares que hayan sido sacrificados después de obtener la licencia, a primeros de abril. Mientras que los embutidos podrán llegar a finales de este año, las paletas de cerdo ibérico no desembarcarán en Estados Unidos hasta 2011, tras un proceso de curación de 18 meses, y los jamones llegarán en 2012, con una curación de 24 meses. Se estima que el jamón ibérico curado podrá alcanzar los 350 dólares por kilo.

Las perspectivas de facturación de COVAP en Estados Unidos prevén alcanzar los 400.000 euros el primer año, un millón de euros en 2011 y 1,5 millones en el siguiente, de forma que en 2013 se puedan alcanzar los 2 millones de euros de ventas anuales con un elevado mar- 
gen, sin tener en cuenta la venta de quesos, que la cooperativa cordobesa inició en el mercado estadounidense hace ya cinco años.

Para alcanzar estas cifras de negocio, la estrategia de ventas tiene previsto iniciar la comercialización por la Costa Este de Estados Unidos, que incluye zonas como Nueva York, Washington DC, Boston o Chicago, además de estados del Noroeste y los de Virginia, Maryland, California y Florida.

En estos mercados, la empresa desarrollará una labor de promoción e información de las bondades del ibérico, su corte, su presentación y su degustación y también contará con el apoyo del ICEX y la Agencia Andaluza de Promoción Exterior-EXTENDA.

Los ibéricos de COVAP se distribuirán a través de "Forever Cheese", que importa y distribuye productos gourmet en 45 de los 50 estados norteamericanos y ya comercializa el queso de la cooperativa en Estados Unidos; "Whole Foods", la cadena líder de supermercados y la más grande del mundo; "Agrosevilla Inc.", una cooperativa sevillana que trabaja con cadenas de pizzerías, y a través de "Tienda.com", que comercializará productos de cerdo ibérico loncheados a través de internet.

Igualmente también se han iniciado las exportaciones a Australia, en este caso de jamones ibéricos deshuesados a través de un importador local.

También en 2010 y para la comercialización del cordero, la cooperativa Covap se ha unido a otras dos cooperativas (Dehesas Cordobesas y San Miguel) para crear una asociación promotora de la marca de calidad Cordero de los Pedroches, una iniciativa que tiene como objetivo la promoción del consumo de este tipo de carne con el marchamo de calidad garantizado en cuanto a origen, sistema productivo y trazabilidad.

Finalmente es de reseñar también la contribución como socio de referencia de la cooperativa COVAP junto otras empresas e instituciones públicas y la Universidad de Córdoba ${ }^{5}$ a la recién creada Fundación CICAP (Centro de Investigación y Calidad Agroalimentaria de Los Pedroches-Córdoba) un centro especializado en $1+D+i$ agroalimentario con el que se quieren desarrollar nuevos productos, procesos de elaboración, envases, entre otros.

La apuesta de futuro de Covap se mantiene en la protección al socio mediante una estrategia permanente de crecimiento y, en palabras de su presidente, Ricardo Delgado, "una forma de insistir en este aspecto es potenciar las ventas en el mercado internacional", de ahí la radical importancia que cobra el proceso de internacionalización de la Cooperativa y

5 En este sentido tal universidad lidera a su vez el proyecto de Campus de Excelencia en Investigación Agroalimentaria cei $_{A} 3$ junto a las universidades de Huelva, Cádiz, Jaén y Almeria que pretende ser un referente en investigación y transferencia entre universidades y tejido productivo. 
Internacionalización de cooperativas agrarias. El modelo de la empresa ... Sánchez C., Sandra M.; Fuentes G., Fernando J. y Santos R., Luna M.

la necesidad de continuar la línea de apertura a nuevos mercados.

\section{Conclusiones}

El fenómeno de la internacionalización se ha convertido en un reto en todos los sectores económicos, abriendo todo un abanico de nuevas posibilidades en el mundo de las cooperativas y así lo entienden las distintas confederaciones y asociaciones que buscan fórmulas de apoyo a sus entidades asociadas para facilitarles el camino.

En el caso de las cooperativas españolas aún se está iniciando el complejo proceso de expansión internacional lo que, a la larga, puede redundar en una mejora de su competitividad y éxito. En el caso de las cooperativas agrarias, aunque la internacionalización aún presenta determinadas barreras estructurales $\mathrm{y}$, sobre todo, de marketing, existe un conjunto de oportunidades que no deben perderse de vista y que les dota de un enorme potencial para la salida a los mercados exteriores.

Pero, aunque se opte por el crecimiento y el proceso de internacionalización para adaptarse a las nuevas exigencias del entorno económico (globalización y disminución de protección agraria comunitaria), las cooperativas no pueden dejar atrás los principios rectores de su propia naturaleza, como son la democracia y la transformación del modelo de empresa y sociedad. Así, las cooperativas internacionalizadas han de enfrentarse al reto de esbozar un nuevo modelo de empresa multinacional cooperativa democrática que, simultáneamente al desarro-
Ilo de políticas socioeconómicas internacionales, mantenga los principios y valores propios de la cooperativa.

Para las cooperativas la estrategia de internacionalización supone un gran reto económico, financiero y social que, aunque no esté exento de riesgos es ineludible en el proceso de creación de un modelo cooperativo multinacional. Asumir este reto es la principal contribución de la sociedad cooperativa al desarrollo económico del futuro. Y, para las cooperativas agrarias, la internacionalización no es ya una opción, sino una exigencia.

La empresa Covap es un buen referente de éxito en la integración estratégica y compromiso de protección al socio y en paralelo de un crecimiento sostenido basado en instalaciones modernas, calidad en todos los procesos y explotación de marcas propias. Con ello se integran las expectativas de todos los grupos de interés: socios-propietarios-proveedores, clientes, trabajadores, directivos y sociedad en general. Esta cooperativa ha conseguido en diez años abrir mercados en 23 países entre los que se encuentran los más exigentes del mundo constituyendo un ejemplo de que es factible la internacionalización de las cooperativas agroalimentarias.

\section{Referencias bibliográficas}

Aranzadi, Dionisio (1999), La empresa cooperativa y sus ventajas competitivas. Estudios Empresariales, LIV, num. 167, San Sebastián.

Baamonde, Eduardo (2009), Cooperativismo agroalimentario. Mediterráneo económico, num. 15, pp.229-246. 
Bajo Rubio, Óscar (1991), Teorías del comercio internacional. Barcelona, Antoni Bosch Editor.

Bakaikoa, Baleren; Errasti, Anjel Mari y Begiristain, Agurtzane (2004), The Governance of MCC. Annals of Public and Cooperative Economics, vol. 75, num. 1, pp. 1-22.

Boccherini, José Antonio (2010), Nuevos retos competitivos para la cadena agroalimentaria española pp. 17-27, en MERCASA (2010), Alimentación en España 2009, Madrid, Ed. Ministerio de Medio Ambiente, Rural y Marino.

Clamp, Christina (2000), The Internationalization of Mondragon. Annals of Public and Cooperative Economics, vol. 71 , num. 4.

Confederación de Cooperativas Agrarias de España CCAE (2010), Observatorio socioeconómico del cooperativismo agroalimentario español. Estadísticas sobre las cooperativas españolas, según datos relativos al ejercicio 2009, Madrid, CCAE.

Confederación General de Cooperativismo Agrario de la UE COGECA (2010), Agricultural cooperatives in Europe: Main issues and trends, Bruselas, COGECA.

Cooperativas Agroalimentarias (2010): Nuestros primeros veinte años. Madrid, Cooperativas Agroalimentarias.

Confederación General de Cooperativismo Agrario de la UE COPA-COGECA (2010), El futuro de la PAC después de 2013. Bruselas, COGECA.

Cornforth, Chris; Thomas, Alan; Lewis, Jenny y Spear, Roger (1988), Developing Successful Worker Co-operatives. Londres, Sage.

Craig, John G. (1976), Multinational Cooperatives. An alternative for world development. Londres, Western Producer Prairie Books: Saskatoon Saskatchewan.
Davies, Keri. y Burt, Steve. (2007), Consumer co-operatives and retail internationalization: problems and prospects. International Journal of Retail and Distribution Management, vol. 35, num. 2, pp. 156-177.

Dicken, Peter (1998), Global Shift, Transforming The World Economy. Londres, Chapman publishing.

Donoso, Ignacio.; Shadbolt, Nicola y Bailey, William (2004). The internationalization of agricultural co-operatives-a source of conflict?, paper presentado en la $14^{\text {th }}$ IAMA World Food and Agribusiness Forum, Symposium and Case Conference, Montreal.

Egerstrom, Lee; Van Dijk, Gert y Bos, Pieter (1996), Seizing Control. The international market power of cooperatives. Rochester: Manchester, Lone Oak Press.

Errasti, Anjel Mari; Heras, Iñaki; Elgoibar, Pilar y Begiristain, Agurtzane (2002), La internacionalización de las cooperativas y su responsabilidad social. Revista de Dirección y Administración de Empresas, núm. 10, pp. 119-145.

Errasti, Anjel Mari; Heras, Iñaki; Bakaikoa, Baleren; Elgoibar, Pilar (2003), The Internationalisationof Cooperatives: The Case of the Mondragon Cooperative Corporation. Annals of Public and Cooperative Economics, vol. 73, num. 4.

Fernández Moreno María del Valle, Peña García-Pardo, Isidoro y Hernández Perlines, Felipe (2008), Factores determinantes del éxito exportador. El papel de la estrategia exportadora en las cooperativas agrarias. CIRIEC, num. 63, pp.39-64.

Federación de Cooperativas Danesas FCD (2000), Transnational co-operatives: perspectives for admission of members abroad and cross-border merg- 
Internacionalización de cooperativas agrarias. El modelo de la empresa ...

Sánchez C., Sandra M.; Fuentes G., Fernando J. y Santos R., Luna M.

ers, disponible en http://www.danskeandelsselskaber.dk/view.asp?ID

=11940, última consulta 21 de enero de 2011.

Geringer, J. Michael; Beamish, Paul W. y Costa, Richard C. da (1989), Diversification strategy and internationalization: implications for MNE performance. Strategic Management Journal, vol. 10 num. 2, pp. 109-119.

Gómez López, José Daniel (2008), El proceso de internacionalización del sector cooperativo agrario: intercooperación y relaciones transnacionales y transfronterizas. V Encontro de Pesquisadores Lation-Americanos de Cooperativismo, Sao Paulo.

Guillén Rodríguez, Mauro F. (2004), La internacionalización de las empresas españolas. Historia Empresarial, num. 812, pp. 211-224.

Hendriske, George W. y Oijen, Aswin C. J. (2002), Diversification and corporate governance (ERIM Report Series ERS-2002-48-ORG), Rotterdam School of Management, Erasmus Universiteit Rotterdam, Rotterdam, Holanda.

Johanson, Jan y Wiedersheim-Paul, Finn (1975), The internationalisation of the firm - four Swedish cases. Journal of Management Studies, vol. 12, num. 3, pp. 305-22.

Juliá Igual, Juan Francisco y Meliá Martí, Elena (2008), Social Economy and the Cooperative movement in Europe: contributions to a new vision of agriculture and rural development in the Europe of the 27. Ciriec, num. 62, pp. 147172.

Kurimoto, Akira (1999), Report on the $9^{\text {th }}$ Symposium of Large Scale Co-operatives in Stockholm, $12^{\text {th }}-14^{\text {th }}$ june, disponible en: www.ccij.co-op.or.jp/ccij/english/ccij-21.htm, última consulta $15 \mathrm{de}$ diciembre de 2010.
Martín, Carlos (2006), Cuatro cooperativas se unen para el fomento de la exportación de porcino. Mundo ganadero, num. 186, pp. 28-31.

Middleton, Alan (1998), Why consumer cooperative societies fail. Journal of Co-operative Studies, vol. 31, num. 2, pp. 3-6.

Mills, Cliff (2002), Distribution and what it means to be a cooperative. Journal of Co-operative Studies, vol. 34, num. 93, pp. 173-181.

Normark, Peter (1996), The Internationalization of Member-Owned Firms. Review of International Cooperation, vol. 89, num. 2, pp. 53-61.

Novkovic, Sonia (2007), R\&D, innovation and networking: strategies for cooperative survival. En Sonia Novkovic \& Vania Sena (Eds.). Cooperative firms in global markets (Vol. 10, pp. 205232). Oxford, Elsevier Ltd.

Pestoff, Víctor (1999), The future of consumer cooperatives in post-industrial societies? Journal of Cooperative Studies, vol. 32, num. 2, pp. 208-219.

Piercy, Nigel (1981), Company Internationalisation: Active and Reactive Exporting. European Journal of Marketing, vol. 15, num. 3, pp. 26- 41.

Ritossa, Claudia Mónica y Bulgacov, Sergio (2009), Internationalization and diversification strategies of agricultural cooperatives: a quantitative study of the agricultural cooperatives in the state of Parana. Brazilian Administration Review, vol. 6, num. 3, pp. 187-212.

Seipel, Michael F. y Heffernan, William D. (1997), The transnational challenge: cooperatives and the global food system. Rural Cooperatives, vol. 64, num. 4, pp. 6-10.

Singerman, Jesse (1987), Why some co-ops die and what to do about the others. Cooperative Grocer, num. 9, febrero-marzo. 
Spear, Roger (2001), Globalisation et stratégies des cooperatives. En Coté, D. (ed), Holdings Cooperatifs: Mutation ou Derive?, pp. 115-138, Bruselas, De Boeck.

Sullivan, Daniel (1994), Measuring the degree of internationalization of a firm. Journal of International Business Studies, vol. 25, num. 2, pp. 325-342.

Theuvsen, Ludwig y Ebneth, Oliver (2005), Internationalization of cooperatives in the agribusiness: concepts of measurement and their application. En Theresia Theurl y Eric Christain
Meyer (Eds). Strategies for Cooperation. pp. 395-419. Alemania, Shaker Verlag $\mathrm{GmbH}$.

Turnbull, Peter W. (1985), Internationalisation of the firm: a stages process or not?. Paper presentado en el Congreso Export Expansion and Market Entry Modes Dalhousie University, Halifax.

Trujillo, María Andrea; Rodríguez, Diego Fernando; Guzmán, Alexander; Becerra, Gisele (2006), Perspectivas teóricas sobre la internalización de empresas. Bogotá D.C., Editorial Universidad del Rosario. 